\title{
Sistemas Inteligentes de Embalagens Utilizando Filmes de Quitosana como Indicador Colorimétrico de Temperatura
}

\author{
Vinícius B. V. Maciel, Telma T. Franco \\ Faculdade de Engenharia Química, UNICAMP \\ Cristiana M. P. Yoshida \\ Faculdade de Engenharia Química, UNICAMP \\ Departamento de Ciências Exatas e da Terra, UNIFESP
}

\begin{abstract}
Resumo: Clorofila, um pigmento natural termossensível, foi incorporado à matriz de filmes de quitosana visando a obter sistemas inteligentes de indicação de variação de temperatura. A quitosana é um polímero biodegradável que forma filmes flexíveis com eficiente barreira ao oxigênio, podendo ser alternativa ao uso de polímeros sintéticos. Clorofila foi adicionada $(0,25 \mathrm{~g} / 100 \mathrm{~g})$ à suspensão de quitosana $(2,00 \mathrm{~g} / 100 \mathrm{~g})$ formando filmes inteligentes de quitosana (FIQ). Os efeitos da temperatura $\left(10{ }^{\circ} \mathrm{C}\right.$ a $\left.50{ }^{\circ} \mathrm{C}\right)$ e luminosidade (0 a $1000 \mathrm{~lx}$ ) foram estudados utilizando um planejamento experimental, avaliando os parâmetros de cor ( $\left.\mathrm{L}^{*}, \mathrm{a}^{*}, \mathrm{~b}^{*}\right)$ e propriedades mecânicas. As suspensões de quitosana contendo clorofila foram aplicadas como revestimento em superfície de papel cartão formando o sistema de material flexível filme-papel cartão (S-FP), reduzindo significativamente o tempo de secagem. Os filmes caracterizaram-se pela homogeneidade, flexibilidade, coloração esverdeada e fácil manuseio. A variação de cor foi visualmente observada no sistema S-FP, alterando irreversivelmente de verde para amarelo quando submetido a temperaturas acima de $50{ }^{\circ} \mathrm{C}$, independentemente da luminosidade. Desta forma, o sistema proposto tem potencial de aplicação como indicador colorimétrico de temperatura na faixa de $50{ }^{\circ} \mathrm{C}$ a $75{ }^{\circ} \mathrm{C}$, com a vantagem de simples fabricação, biodegradabilidade e uso de materiais seguros para aplicação em contato direto com alimentos e fármacos, além do baixo custo.
\end{abstract}

Palavras-chave: Embalagem inteligente, quitosana, clorofila, indicadores de temperatura.

\section{Alternative Intelligent Material for Packaging Using Chitosan Films as Colorimetric Temperature Indicators}

\begin{abstract}
Chlorophyll was incorporated into chitosan films, forming intelligent systems able to detect variations in temperature. Chitosan is a biodegradable polymer that forms flexible, resistant films with an efficient oxygen barrier. Chlorophyll was added $(0.25 \mathrm{~g} / 100 \mathrm{~g})$ into chitosan suspension $(2.00 \mathrm{~g} / 100 \mathrm{~g})$ and intelligent films (FIQ) were cast. The effects of temperature $\left(10^{\circ} \mathrm{C}\right.$ to $\left.50^{\circ} \mathrm{C}\right)$ and luminosity $(0$ to $1000 \mathrm{~lx})$ on the films were studied using an experimental design with two variables, analyzing the color parameters $\mathrm{L}^{*}$, $\mathrm{a}^{*}, \mathrm{~b}^{*}$ and mechanical properties. The films were characterized according to their homogeneity, flexibility, color and handling. Chitosan suspensions containing chlorophyll were also applied as coating on the surface of pieces of card-paper, forming a card paper film system (S-FP). By visual inspection we observed the color of the S-FP changing irreversibly from green to yellow when the temperature was above $50{ }^{\circ} \mathrm{C}$. Therefore, the S-FP system can potentially be used as a colorimetric indicator for the $50{ }^{\circ} \mathrm{C}$ to $70{ }^{\circ} \mathrm{C}$ range, being advantageous owing to the ease of fabrication, biodegradability, safe use for food and low cost.
\end{abstract}

Keywords: Intelligent packaging, chitosan, chlorophyll, temperature indicator.

\section{Introdução}

O potencial de aplicação de novos sistemas de acondicionamento vem aumentando devido às exigências do consumidor e preocupação sobre a procedência e condições de transporte e armazenamento. A rastreabilidade é uma ferramenta utilizada para garantir a segurança e qualidade do produto; no caso de alimentos, a segurança alimentar e para fármacos, a ação do composto bioativo. As indústrias de embalagens estão buscando novos métodos objetivos, realizáveis e de custo efetivo, que mantenham a integridade do produto, visando garantir qualidade e segurança ${ }^{[1]}$, e a confiança do consumidor ${ }^{[2]}$.

Embalagens inteligentes consistem em sistemas onde é possível a comunicação com o consumidor, de informações relacionadas à qualidade e segurança do produto através da embalagem. Isto envolve a incorporação de sensores ou indicadores que respondem por sinal (elétrico, colorimétrico, etc.) às alterações das condições iniciais $^{[3]}$.
A utilização de polímeros naturais na formação de embalagens flexíveis vem sendo intensamente estudada em âmbito mundial, visando substituição parcial dos polímeros sintéticos, principalmente devido ao problema ambiental de acúmulo de lixo de difícil degradação ${ }^{[2]}$. Dentre os polímeros naturais, a quitosana tem despertado interesse, pois é biodegradável, provém de fontes renováveis e é capaz de formar filmes flexíveis e resistentes, com barreira eficiente a oxigênio, além de atividade antimicrobiana ${ }^{[4,5]}$.

Nesta última década, inúmeros estudos foram desenvolvidos visando a produção e caracterização de filmes biodegradáveis à base de macromoléculas naturais, como proteínas de soro de leite $^{[6,7]}$, gelatina ${ }^{[8-10]}$, zeína ${ }^{[11-13]}$, quitosana ${ }^{[14-17]}$, quinoa ${ }^{[18]}$, amido de mandioca ${ }^{[19-22]}$, proteína de $\operatorname{soja}^{[23]}$, pectina ${ }^{[24]}$, alginato ${ }^{[25-26]}$, entre outros. Entretanto, a abordagem de aplicação destes materiais como sensores em embalagens inteligentes, ainda é escassa na literatura especializada. 
Entre os sensores utilizados em embalagens de alimentos, encontram-se aqueles que informam variações na temperatura. Esta é uma das variáveis mais importantes que influencia a qualidade e segurança de produtos alimentícios e fármacos durante a distribuição e estocagem. Uma embalagem adequada promove barreira efetiva contra gases e umidade, mas a temperatura depende de condições de distribuição e estocagem. Monitorar a temperatura pode favorecer o estudo mais preciso de vida de prateleira dos produtos corrigindo o período de validade, aumentando o controle de qualidade e reduzindo o desperdício dos produtos ${ }^{[27]}$. A grande extensão territorial brasileira associada ao transporte de cargas predominantemente rodoviário promove situações adversas prejudiciais à manutenção da segurança e qualidade dos produtos. Assim, um dispositivo informando variações de temperatura seria importante. Indicadores tempo-temperatura são definidos como dispositivos simples e de custo efetivo utilizados como sistemas de embalagens inteligentes para monitorar, registrar e indicar qualquer alteração de temperatura a que produtos possam ter sido expostos desde a fabricação até o transporte e armazenamento. Exemplos destes sistemas já são encontrados comercialmente ${ }^{[28-29]}$, porém utilizam materiais sintéticos ou precisam de dispositivos eletrônicos como a tecnologia RFID (Radio Frequency Identification).

Alguns pigmentos naturais são termossensíveis, ou seja, ocorrem alterações em sua estrutura e cor quando expostos ao calor $^{[30]}$. Exemplos desses tipos de pigmentos são: betalaínas, urucum, antocianinas, pigmento de Monascus, clorofilas, carmim de cochonilha e curcumina. Para o caso de embalagens em contato direto com o alimento ou produto farmacêutico, todos os pigmentos citados acima são permitidos pela legislação brasileira de acordo com Resolução n. 44 da Agência Nacional de Vigilância Sanitária ${ }^{[31]}$.

O objetivo deste trabalho foi a obtenção de um novo tipo de indicador de temperatura, de simples fabricação, obtido a partir de material biodegradável e proveniente de fonte natural, utilizando filmes de quitosana contendo clorofila aplicados como revestimento em papel cartão. A eficiência desse sistema foi analisada por alteração de cor pelos parâmetros L*, a*, b*.

\section{Material e Métodos}

\section{Material}

Quitosana (Primex, grau de acetilação $18 \%$ e massa molecular $2,38 \times 10^{5}$ g.mol ${ }^{-1}$, Islândia), ácido acético (Synth, Brasil), clorofila (Christian Hansen, C-10000-WS-P, Brasil) e papel cartão triplex TP 250 g. $\mathrm{m}^{-2}$ (Suzano Papel e Celulose Ltd., Brasil).

\section{Métodos}

\section{Filmes inteligentes de quitosana (FIQ)}

A metodologia de produção foi adaptada de Yoshida et al. $(2009)^{[15]}$. Os filmes foram produzidos por via úmida, solubilizando 2,00 g/100 g de suspensão filmogênica de quitosana, contendo o indicador colorimétrico clorofila na concentração de $0,25 \mathrm{~g} / 100 \mathrm{~g}$. Ácido acético foi adicionado estequiometricamente, de acordo com o grau de acetilação e massa de quitosana, para promover a protonação dos grupos $\mathrm{NH}_{2}{ }^{[32]}$. A suspensão foi mantida sob agitação contínua, em agitador magnético, por 45 minutos, até solubilização total. Em seguida, foi dispersa em suporte plano (placas de Petri). A espessura dos filmes foi mantida constante controlando-se a relação massa/área $\left(\mathrm{g} . \mathrm{m}^{-2}\right)$ aplicada. A etapa seguinte correspondeu à secagem dos filmes, realizada em duas fases: secagem a temperatura ambiente $\left(25^{\circ} \pm 1^{\circ} \mathrm{C}\right)$ por 36 horas e posteriormente em estufa com circulação forçada de ar a $28{ }^{\circ} \mathrm{C}$ por 24 horas. O conteúdo final de sólidos totais nos filmes secos foi $28,2 \mathrm{~g} \cdot \mathrm{m}^{-2}$.

\section{Planejamento experimental}

A estabilidade de cor dos filmes FIQ foi determinada para mudanças de temperatura e luminosidade em função do tempo de armazenamento. Um planejamento experimental fatorial de dois níveis foi utilizado para estudar os efeitos individuais e combinados das variáveis temperatura e luminosidade (Tabela 1).

A intensidade luminosa na incubadora foi controlada utilizando lâmpadas com diferentes potências (W), sem interferência de luz ambiente, calculando a luminosidade em função da área. A faixa escolhida de luminosidade foi baseada em uma condição de ausência de luz (0 lx), valores padronizados de iluminação geral para área de trabalho (500 lx), e simulando exposição em supermercado (1000 lx), baseada nos padrões da norma NBR 5413 $3^{[33]}$. A temperatura foi controlada utilizando incubadora BOD (Tecnal, TE-391, Brasil) com circulação forçada de ar. As respostas analisadas foram os parâmetros de cor $\left(\mathrm{L}^{*}, \mathrm{~h}_{\mathrm{ab}}\right)$ e as propriedades mecânicas dos filmes (deformação, tensão de ruptura e módulo de rigidez), sendo definido o tempo de 72 horas para avaliar a influência das variáveis temperatura e luminosidade nos filmes FIQ.

\section{Avaliação da cor}

A avaliação de cor foi realizada usando colorímetro Chroma Meter CR 400 (Konica Minolta, Japão) nas amostras de FIQ submetidas a diferentes temperaturas e luminosidades. As medidas foram realizadas no sistema CIE $L^{*} a^{*} b^{*}$. O parâmetro $L^{*}$ representa o quanto uma amostra é clara ou escura e varia em escala de 0 (preto) a 100 (branco), a* é a medida verde/vermelho e b* é a medida na faixa azul/amarelo. Ambas escalas $a^{*} \mathrm{e} \mathrm{b}^{*}$ variam de $-60 \mathrm{a}+60$. Para avaliação dos parâmetros de cor $\mathrm{a}^{*} \mathrm{e} \mathrm{b}^{*}$ pelo planejamento experimental, foi necessário, de acordo com Wyszecki e Stiles (1967) ${ }^{[34]}$ e Gonçalves et al. (2009) ${ }^{[35]}$ a transformação destes parâmetros em valores geométricos, sendo representado por hue angle $\left(\mathrm{h}_{\mathrm{ab}}\right) . \mathrm{O}_{\mathrm{ab}}$ é calculado pelo arctan $\mathrm{b} * / \mathrm{a}^{*}$. Os dados foram obtidos em triplicata.

Uma avaliação da alteração de cor também foi obtida em função do tempo nos filmes FIQ, sendo realizada a partir dos resultados dos parâmetros de cor ( $\left.\mathrm{L}^{*}, \mathrm{a}^{*}, \mathrm{~b}^{*}\right)$, nos tempos $0,12,24,48 \mathrm{e}$ 72 horas, em incubadora (Tecnal, TE-391, Brasil) com temperatura e luminosidade controlada, conforme condições propostas nos ensaios do planejamento experimental.

\section{Propriedades mecânicas}

A deformação $(\varepsilon)$, tensão na ruptura $(\operatorname{Tr})$ e módulo de rigidez (E) foram determinados nas amostras de filmes FIQ de acordo com o método ASTM D882 ${ }^{[36]}$. Os filmes FIQ foram cortados em dimensões de $25,4 \times 100,0 \mathrm{~mm}$ e pré-acondicionados a $50 \%$ de umidade relativa e $25 \pm 2{ }^{\circ} \mathrm{C}$ por 48 horas. As medidas foram realizadas utilizando-se texturômetro TA.XT2 (TA Instruments, Inglaterra) com distância inicial de separação fixada em $50 \mathrm{~mm}$ e velocidade de realização do teste igual a $1,0 \mathrm{~mm} / \mathrm{s}$. Para a realização desses testes, até dezoito filmes foram utilizados. A espessura foi medida em micrômetro MDC-25M (Mitutoyo, Japão), com cinco leituras aleatórias utilizando a média dos valores para calcular as propriedades dos filmes.

\section{Sistema filme-papel cartão (S-FP)}

Folhas de papel cartão $\left(0,045 \mathrm{~m}^{2}\right)$ foram revestidas com a suspensão de filmes FIQ. As concentrações de quitosana e clorofila

Tabela 1. Faixa de valores utilizados no planejamento fatorial $2^{2}$.

\begin{tabular}{cccc}
\hline Variáveis & $\mathbf{- 1}$ & $\mathbf{0}$ & $\mathbf{+ 1}$ \\
\hline Temperatura $\left({ }^{\circ} \mathrm{C}\right)$ & 10 & 30 & 50 \\
Luminosidade $(\mathrm{lx})$ & 0 & 500 & 1000 \\
\hline
\end{tabular}


usadas foram de 2,00 g/100 g e $0,25 \mathrm{~g} / 100 \mathrm{~g}$, respectivamente, obtendo o conteúdo final de sólidos totais no revestimento igual a $0,081 \mathrm{~g} \cdot \mathrm{m}^{-2}$. A suspensão de FIQ foi espalhada na superfície do papel cartão usando barra de $80 \mu \mathrm{m}$ (TKB Erichsen, Brasil). O $\mathrm{S}-\mathrm{FP}$ foi seco a $150^{\circ} \mathrm{C}$ por 90 segundos em estufa adaptada modelo 315 SE (FANEM, Brasil).

A alteração de cor para o sistema S-FP foi avaliada em função dos parâmetros $\mathrm{L}^{*}, \mathrm{a}^{*}, \mathrm{~b}^{*}$ obtidos por leitura em colorímetro Chroma Meter CR 400 (Konica Minolta, Japão) após 72 horas de exposição em diferentes temperaturas $\left(50^{\circ}, 60^{\circ}\right.$ e $\left.75^{\circ} \mathrm{C}\right)$ e luminosidades (0 e 1000 1x), utilizando-se incubadora (Tecnal, TE-391, Brasil) com controle de temperatura e luminosidade. O controle foi adotado como a medida de amostras de papel revestido no tempo zero na condição de $25^{\circ} \mathrm{C}$.

\section{Microscopia eletrônica de varredura (MEV)}

A microestrutura do sistema S-FP foi avaliada por análise de MEV, utilizando-se microscópio Leica (Inglaterra) modelo LEO440i. As amostras foram previamente acondicionadas em dessecador contendo sílica gel durante 48 horas à $25^{\circ} \mathrm{C}$ para posteriormente serem fixadas em suportes (stubs) de alumínio para a metalização em ouro. A metalização foi realizada em equipamento VG Microtech (Inglaterra) modelo SC7620 sputter coater durante 180 segundos a $4 \mathrm{~mA}$. As imagens foram analisadas na superfície e secção transversal do sistema S-FP. Folhas de papel cartão sem revestimento foram estudados como referência.

\section{Análise estatística}

O programa Statistic 7.0 (Statistica, EUA) foi utilizado para análises estatísticas aplicadas no planejamento experimental ( $\mathrm{p} \leq 0,1)$, análise de variância (ANOVA) e teste de Tukey com nível de significância $\mathrm{p} \leq 0,05$.

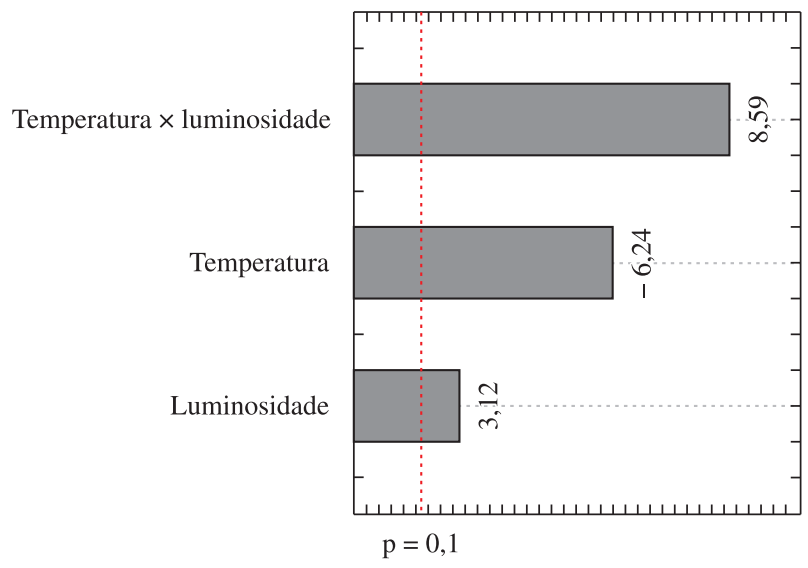

(a)

\section{Resultados e Discussão}

\section{Filmes FIQ: planejamento experimental}

Os filmes FIQ caracterizaram-se pela homogeneidade, flexibilidade, transparência, coloração verde brilhante e fácil manuseabilidade.

Os parâmetros de cor $L^{*}, h_{a b}$ e as propriedades mecânicas das amostras de FIQ foram avaliados após 72 horas de exposição em diferentes condições de temperatura e luminosidade (Tabela 2).

Os efeitos da temperatura e luminosidade na alteração de cor ( $\mathrm{L}^{*}$ e $\mathrm{h}_{\mathrm{ab}}$ ) dos filmes FIQ foram significativos para um nível de confiança de $90 \%$ (Figura 1).

$\mathrm{O}$ aumento da temperatura de $10{ }^{\circ} \mathrm{C}$ para $50{ }^{\circ} \mathrm{C}$ provocou uma redução no parâmetro $L^{*}$ na ordem de 6,24 , ou seja, os filmes tenderam a apresentar coloração mais escura. E o aumento da luminosidade de 0 para 1000 lx e da combinação temperatura/luminosidade aumentaram os valores de L* em 3,12 e 8,59, respectivamente, tendendo à descoloração dos filmes FIQ.

Apenas a luminosidade promoveu efeito significativo no parâmetro $h_{a b}$, observando que o aumento de 0 para 1000 lx, reduziu os valores na ordem de 2,92, tendendo a coloração mais amarela dos filmes FIQ.

Apesar da variação numérica dos parâmetros de cor, não foi possível visualizar a mudança nos filmes FIQ. Este fenômeno foi associado à alta concentração de sólidos totais nos filmes FIQ e também ao uso de clorofilina ao invés da clorofila. A molécula de clorofila (Figura 2) é transformada em clorofilina cúprica (uma espécie de clorofila solúvel em água) pela substituição do seu átomo de magnésio central pelo íon $\mathrm{Cu}^{2+}$, o que promove maior estabilidade da molécula, dificultando a perda de cor na presença de luminosidade e variações de temperatura ${ }^{[37,38]}$. Muitas indústrias de

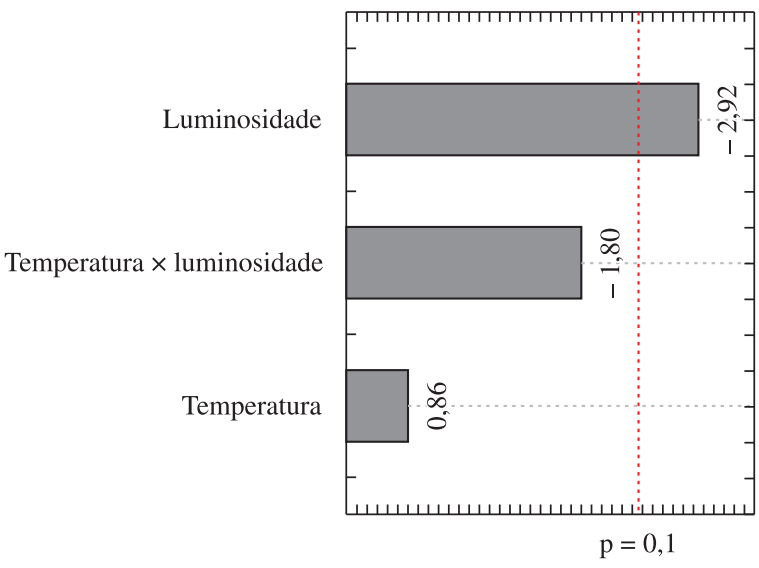

(b)

Figura 1. Efeitos da temperatura e luminosidade na alteração de cor dos filmes FIQ: (a) parâmetro L* e (b) parâmetro $h_{a b}$.

Tabela 2. Matriz do planejamento fatorial $2^{2}$ para os filmes FIQ e as respostas em parâmetros de cor e propriedades mecânicas.

\begin{tabular}{|c|c|c|c|c|c|c|c|}
\hline \multirow{3}{*}{ Ensaios } & \multicolumn{2}{|c|}{ Variáveis independentes } & \multicolumn{2}{|c|}{ Cor } & \multicolumn{3}{|c|}{ Propriedades mecânicas } \\
\hline & Temperatura & Luminosidade & \multirow{2}{*}{$\mathbf{L}^{*}$} & \multirow{2}{*}{$\mathbf{h}_{\mathrm{ab}}\left({ }^{\circ}\right)$} & $\varepsilon$ & Tr & $\mathbf{E}$ \\
\hline & $\left({ }^{\circ} \mathbf{C}\right)$ & (lx) & & & $(\%)$ & (MPa) & (GPa) \\
\hline 1 & $-1(10)$ & $-1(0)$ & 58,49 & 131,92 & 3,73 & 57,79 & 3,15 \\
\hline 2 & $+1(50)$ & $-1(0)$ & 54,64 & 132,58 & 4,09 & 64,98 & 3,11 \\
\hline 3 & $-1(10)$ & $+1(1000)$ & 57,07 & 131,53 & 4,22 & 56,07 & 3,08 \\
\hline 4 & $+1(50)$ & $+1(1000)$ & 57,68 & 130,93 & 4,13 & 64,07 & 3,15 \\
\hline 5 & $0(30)$ & $0(500)$ & 56,51 & 131,60 & 2,49 & 64,59 & 3,24 \\
\hline 6 & $0(30)$ & $0(500)$ & 56,86 & 131,43 & 2,46 & 64,06 & 3,25 \\
\hline 7 & $0(30)$ & $0(500)$ & 57,07 & 131,09 & 2,46 & 64,45 & 3,27 \\
\hline
\end{tabular}


alimentos vêm utilizando clorofilina ao invés de clorofila em seus produtos devido à sua maior estabilidade.

Os efeitos da temperatura e luminosidade também foram avaliados para as propriedades mecânicas deformação $(\varepsilon)$, tensão na ruptura (Tr) e módulo de rigidez (E) dos filmes FIQ (Tabela 2).

Para $\varepsilon$ e E, a temperatura, luminosidade e combinação entre elas, não influenciaram significativamente $(\mathrm{p} \leq 0,1)$ dentro da faixa de temperatura e luminosidade estudada. Para $\mathrm{Tr}$ apenas a temperatura teve efeito significativo, observando um aumento na ordem de $2,74 \mathrm{MPa}$, ao aumentar a temperatura de $10{ }^{\circ} \mathrm{C}$ para $50{ }^{\circ} \mathrm{C}$, independente da presença ou ausência de luminosidade.

A influência da temperatura sobre a Tr, pode estar associado à evaporação do solvente da matriz dos filmes FIQ com o aumento da temperatura. Moléculas de água podem atuar como agente plastificante dentro da matriz filmogênica de polímeros naturais e, sua eliminação, pode influenciar diretamente nas propriedades mecânicas finais, reduzindo a flexibilidade e aumentando a resistência ${ }^{[6]}$.

\section{Tempo de resposta colorimétrica}

FIQ

Para avaliar o tempo de resposta deste novo sistema indicador de temperatura, os filmes FIQ foram expostos nas mesmas condições do planejamento experimental e a alteração da cor foi medida pelos parâmetros $L^{*}, a^{*}, b^{*}$ durante $0,12,24,48$ e 72 horas. $\mathrm{O}$ parâmetro $\mathrm{L}^{*}$ não alterou significativamente nos filmes FIQ durante este período. Olarte et al. (2009) ${ }^{[39]}$ estudando os efeitos da

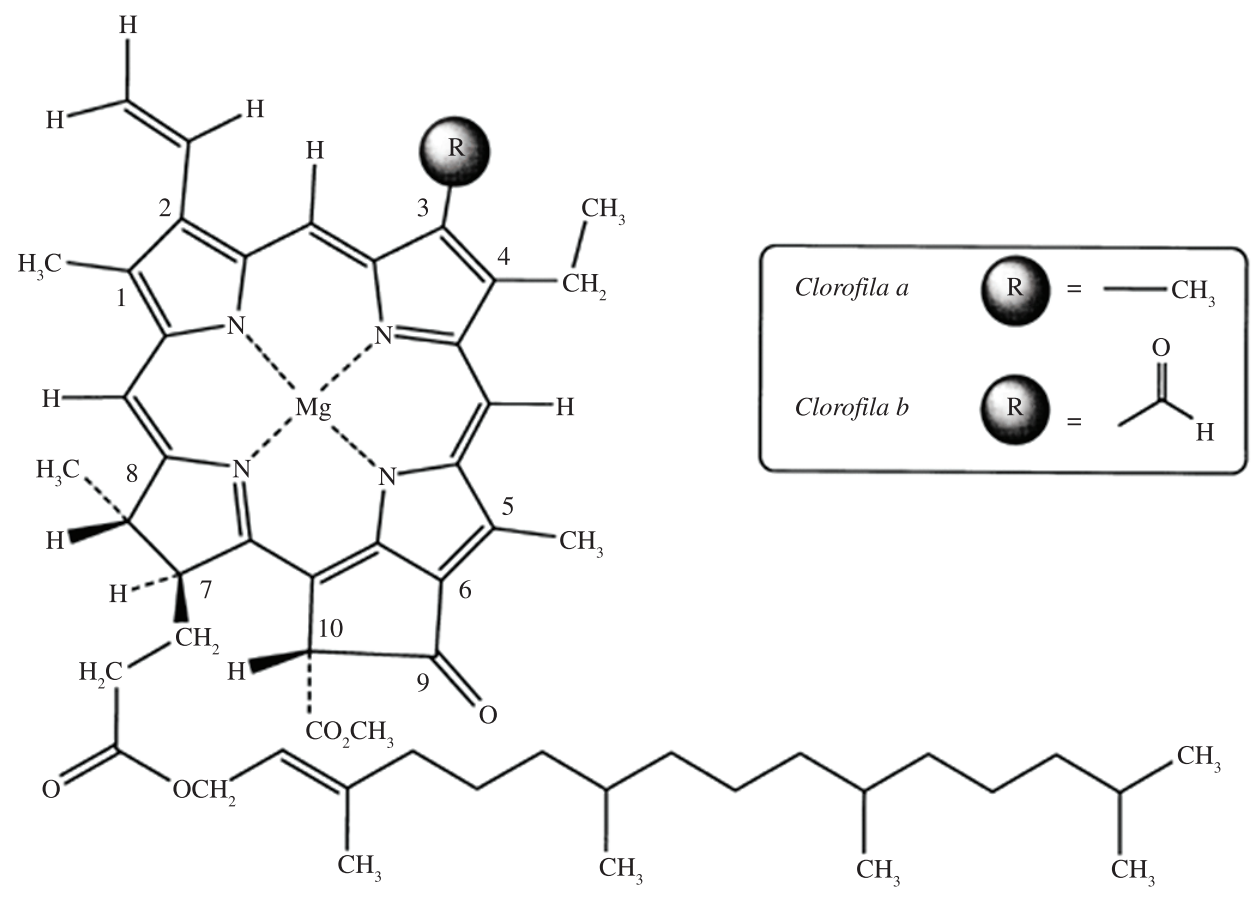

Figura 2. Estrutura química da clorofila ${ }^{[37]}$.

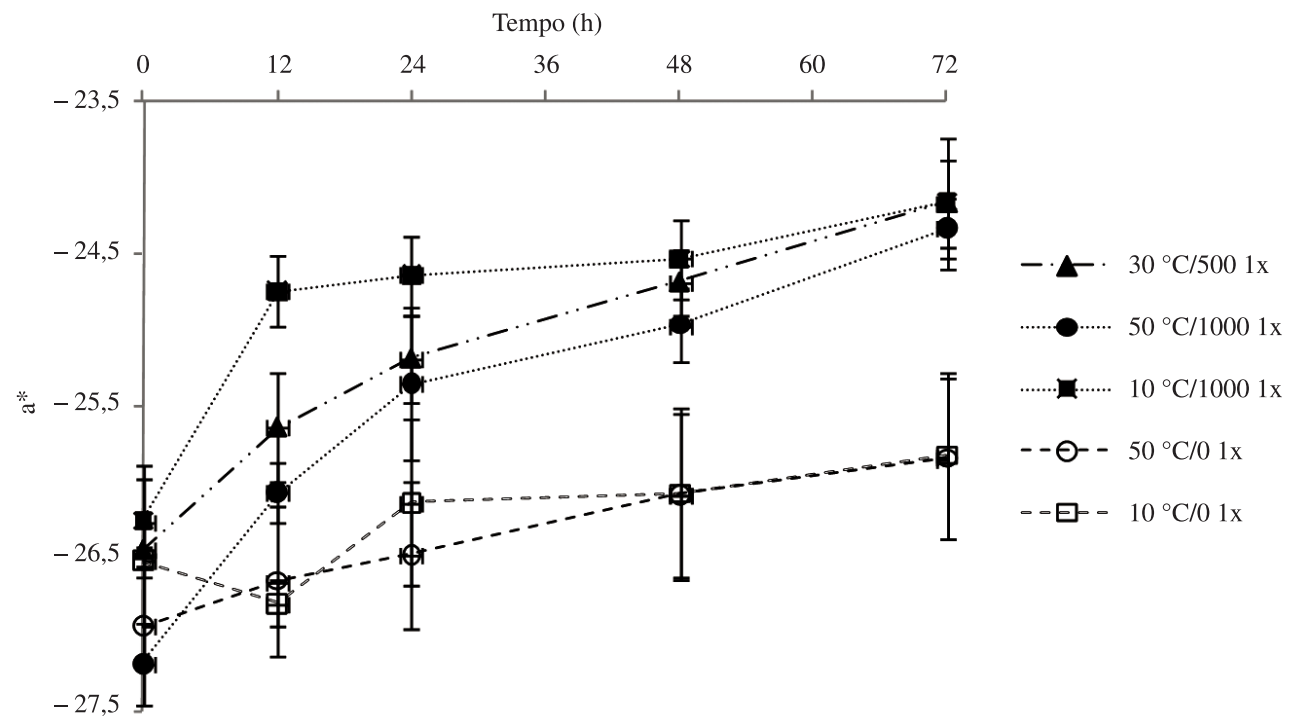

Figura 3. Parâmetro de cor a* dos filmes FIQ, durante 72 horas de exposição em diferentes condições de temperaturas e luminosidade. 
luminosidade durante a estocagem de brócolis e couve encontraram que o valor de $\mathrm{L}^{*}$ diminuiu com o tempo de armazenamento sob efeito da luminosidade. Outros pesquisadores também verificaram que brócolis quando exposto à luminosidade por longos períodos tenderam a coloração amarela, perdendo a cor original verde brilhante ${ }^{[40]}$.

Com 12 horas de exposição em diferentes temperaturas e 500 e 1000 lx, o parâmetro a* dos filmes FIQ apresentou variação significativa da cor, com aumento na faixa de 8 a $11 \%$, caracterizado pela alteração de cor para vermelho. Após este período, os filmes FIQ tenderam à estabilidade (Figura 3 ).

$\mathrm{Na}$ ausência de luminosidade (0 1x), o aumento da temperatura de $10{ }^{\circ} \mathrm{C}$ para $50{ }^{\circ} \mathrm{C}$ não provocou alteração significativa de cor nos filmes.

$\mathrm{O}$ parâmetro $b^{*}$ diminuiu significativamente $(\mathrm{p} \leq 0,05)$ quando se manteve a temperatura constante em $10{ }^{\circ} \mathrm{C}$ e aumentou a luminosidade de 0 para $1000 \mathrm{~lx}$. Os filmes FIQ expostos à $50^{\circ} \mathrm{C}$, e submetidos à $0 \mathrm{~lx}$ ou $1000 \mathrm{~lx}$ não apresentaram variações significativas, indicando que a luminosidade não influenciou na alteração de cor nesta temperatura. Esta alteração de cor observada, foi explicada pela conversão da clorofila em feofitinas, que ocorre

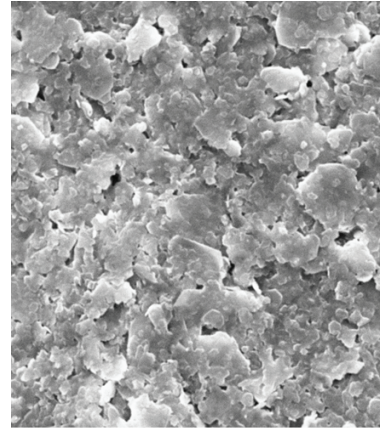

$2 \overline{\mu m}$

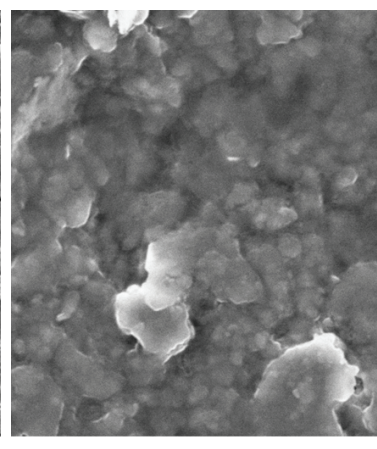

$2 \overline{\mu \mathrm{m}}$
Mag $=5.00 \mathrm{k} \mathrm{x}$

(b)

(a)

Figura 4. Micrografias obtidas por MEV da região superficial do sistema S-FP para: (a) papel sem revestimento; (b) revestido com suspensão de filmes FIQ.

Tabela 3. Parâmetros de cor do sistema S-FP após 72 horas de exposição em diferentes condições de temperaturas e luminosidades.

\begin{tabular}{cccc}
\hline Condições & Parâmetro L* & Parâmetro a* & Parâmetro b* \\
\hline Controle & $84,59 \pm 0,26^{\mathrm{a}}$ & $-3,42 \pm 0,26^{\mathrm{a}}$ & $9,65 \pm 0,28^{\mathrm{a}}$ \\
$50{ }^{\circ} \mathrm{C} / 1000 \mathrm{~lx}$ & $84,86 \pm 0,21^{\mathrm{a}}$ & $-2,51 \pm 0,07^{\mathrm{b}}$ & $10,24 \pm 0,22^{\mathrm{b}}$ \\
$60^{\circ} \mathrm{C} / 01 \mathrm{x}$ & $84,53 \pm 0,13^{\mathrm{a}}$ & $-3,19 \pm 0,07^{\mathrm{ac}}$ & $9,79 \pm 0,11^{\mathrm{ab}}$ \\
$75^{\circ} \mathrm{C} / 01 \mathrm{x}$ & $84,23 \pm 0,04^{\mathrm{a}}$ & $-2,96 \pm 0,11^{\mathrm{c}}$ & $11,79 \pm 0,15^{\mathrm{c}}$ \\
\hline
\end{tabular}

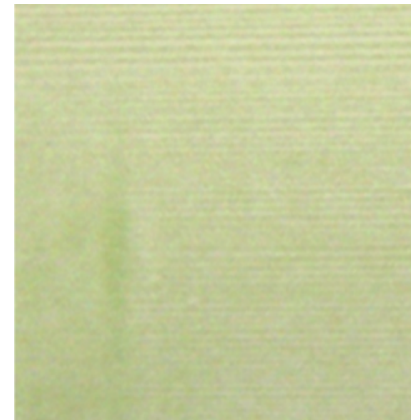

(a)

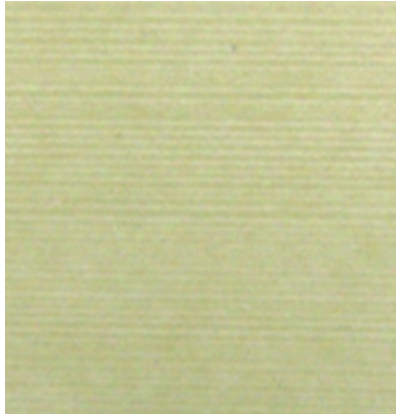

(b)

quando a clorofila perde seu átomo de magnésio e ganha um hidrogênio, apresentando coloração verde-oliva ${ }^{[41]}$.

Apesar das alterações medidas no colorímetro, os filmes FIQ não apresentaram modificações visualmente perceptíveis. Resultados semelhantes foram encontrados por Gonçalves et al. $(2009)^{[35]}$ que pesquisando a cinética de degradação de brócolis antes e após o branqueamento $\left(70-90{ }^{\circ} \mathrm{C}\right)$ verificaram que apesar das pequenas alterações nos parâmetros de cor, o vegetal continuou com sua cor verde brilhante, não como a inicial, mas sem mudanças visuais significativas.

\section{Sistema filme-papel cartão (S-FP)}

As suspensões dos filmes FIQ foram aplicadas como revestimento em folhas de papel cartão, o que reduziu efetivamente o período de secagem. Os filmes FIQ foram obtidos após 60 horas de secagem, e aplicando-os como revestimento em papel cartão, o período de secagem reduziu para 90 segundos. Este é um fator importante na tecnologia de fabricação, pois facilita a produção e comercialização em grande escala. Os filmes FIQ apresentaram boa aderência à superfície do papel cartão, obtendo-se um sistema de coloração esverdeada, homogêneo, sem poros ou defeitos (Figura 4). O manuseio do sistema, promovendo dobras e desgastes, não provocou a delaminação do revestimento.

As imagens da secção transversal do sistema S-FP não apresentaram diferença para as imagens do papel sem revestimento, indicando que a quantidade de sólidos totais na superfície foi absorvida pelas fibras de celulose. Segundo Fernandez et al. $(2010)^{[42]}$, a absorção de quitosana entre as fibras de celulose das folhas de papel ocorrem progressivamente atingindo a saturação após três camadas de revestimento da suspensão polimérica.

Os sistemas S-FP apresentaram variação de cor visível e irreversível após exposição em diferentes condições de temperatura, na presença e ausência de luminosidade $\left(50^{\circ} \mathrm{C} / 1000 \mathrm{~lx}, 60^{\circ} \mathrm{C} / 0 \mathrm{~lx}\right.$ e $75^{\circ} \mathrm{C} / 1000 \mathrm{~lx}$ ). Um resultado contrário ao encontrado nos filmes FIQ, o que pode estar associado a uma menor quantidade de sólidos totais depositados na superfície do papel, favorecendo a exposição da molécula de clorofilina (Figura 5).

$\mathrm{Na}$ ausência de luminosidade $(0 \mathrm{~lx})$, quanto maior a temperatura de exposição, maior a mudança da cor verde para amarelo. E a combinação de maiores temperatura e luminosidade $\left(50^{\circ} \mathrm{C} / 1000 \mathrm{~lx}\right)$ também promoveu a tendência de alterar a cor do sistema para amarelo. Estas mudanças no sistema S-FP foram medidas pelos parâmetros de cor $\left(L^{*}, a^{*}\right.$ e $\left.b^{*}\right)$ apresentados na Tabela 3.

A luminosidade acelerou o processo de alteração de cor, pois ao submeter o sistema à $50^{\circ} \mathrm{C} / 1000 \mathrm{~lx}$ houve mudança estatisticamente significativa nos parâmetros $a^{*}$ e b*, onde o sistema apresentou tendência a perder coloração verde adquirindo tons amarelos.

$\mathrm{Na}$ ausência de luminosidade $(0 \mathrm{~lx})$, ao aumentar a temperatura para $60{ }^{\circ} \mathrm{C}$, o sistema não apresentou alteração significativa quando comparada à condição controle. E após a exposição a $75^{\circ} \mathrm{C}$, somente

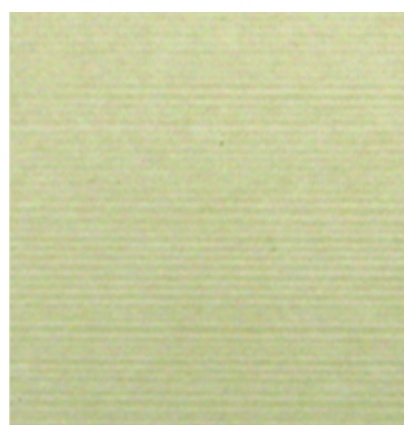

(c)

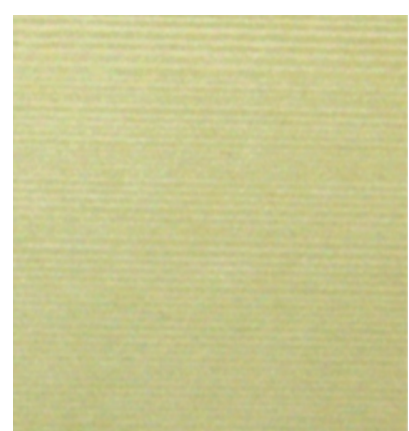

(d)

Figura 5. Variação de cor dos sistemas S-FP expostos em diferentes condições (a) Controle; (b) $50{ }^{\circ} \mathrm{C} / 1000$ 1x; (c) $60{ }^{\circ} \mathrm{C} / 0$ lx e (d) $75{ }^{\circ} \mathrm{C} / 0$ lx. 
o parâmetro b* apresentou alteração significativa, representado pela tendência à coloração amarela.

A mudança de cor pode estar associada à degradação da clorofilina, que sob elevadas temperaturas e presença de luminosidade pode se formar compostos de coloração marrom, denominados feoforbídeos ${ }^{[43]}$.

\section{Conclusão}

Um novo material de embalagem com potencial de aplicação como indicador colorimétrico de temperatura específico para a faixa de $50{ }^{\circ} \mathrm{C}$ a $75{ }^{\circ} \mathrm{C}$ foi obtido a partir de filmes de quitosana contendo clorofila aplicados como revestimento em papel cartão. $\mathrm{O}$ sistema altera irreversivelmente de verde para amarelo quando exposto nesta faixa de temperatura. As vantagens deste sistema são a biodegradabilidade, simples fabricação e ser constituído por materiais naturais e seguros para uso em contato com alimentos. O potencial de aplicação deste sistema é no setor de embalagens, podendo informar diretamente aos consumidores, as reais condições que produtos termossensíveis, como alimentos, fármacos, produtos biológicos foram submetidos durante a cadeia de transporte e estocagem. O Brasil é um país de grande extensão territorial e o transporte ainda é predominantemente rodoviário, o que torna o controle da temperatura um fator de grande importância na garantia da qualidade e segurança do produto embalado.

\section{Agradecimentos}

Os autores agradecem à CAPES, CNPq, FAPESP e SUZANO Papéis e Celulose, pelo apoio no desenvolvimento deste trabalho.

\section{Referências Bibliográficas}

1. Ouattara, B.; Simard, R. E.; Piette, G.; Begin, A. \& Holley, R. A. - J. Food Sci., 65, p.768 (2000). http://dx.doi.org/10.1111/j.1365-2621.2000. tb13584. $\mathrm{x}$

2. Aider, M. - Food Sci. Technol., 43, p.837 (2010).

3. Dainelli, D.; Gontard, N.; Spyropoulos, D.; Zondervan, E. \& Tobback, P. - Trends Food Sci. Technol., 19, p.S103 (2008). Pmid:21299575. http://dx.doi.org/10.1016/j.tifs.2008.09.011

4. Torres, M. A.; Vieira, R. S.; Beppu, M. M. \& Santana, C. C. Polímeros, 15, n.4, p.306-312, 2005. http://dx.doi.org/10.1590/ S0104-14282005000400016

5. Chen, X. G.; Zheng, L.; Wang, Z.; Lee, C. Y. \& Park, H. J. - J. Agr. Food Chem., 50, p.5915 (2002). PMid:12358459. http://dx.doi.org/10.1021/ jf020151g

6. Yoshida, C. M. P. \& Antunes, A. J. - Ci. Tecnol. Aliment., 29, p.420 (2009). http://dx.doi.org/10.1590/S0101-20612009000200030

7. Zinoviadou, K. G.; Koutsoumanis, K. P. \& Biliaderis, C. G. - Food hydrocoll., 24, p.49 (2010).

8. Carvalho, R. A. \& Grosso, C. R. F. - Braz. J. Chem. Eng., 23, p.45 (2006).

9. Fakhouri, F. M.; Fontes, L. C. B.; Gonçalves, P. V. M.; Milanez, C. R.; Steel, C. J. \& Collares, F. P. - Ciên. Tecnol. Aliment., 27, p.369 (2007). http://dx.doi.org/10.1590/S0101-20612007000200027

10. Wang, L.; Auty, M. A. E. \& Kerry, J. P. - J. Food Eng., 96, p.199 (2010). http://dx.doi.org/10.1016/j.jfoodeng.2009.07.025

11. Shi, K.; Kokini, J. \& Huang, Q. - J. Agr. Food Chem., 57, p.2186 (2009). PMid:19231898. http://dx.doi.org/10.1021/jf803559v

12. Tihminlioglu, F.; Atik, I. D. \& Özen, B. - J. Food Eng., 96, p.342 (2010). http://dx.doi.org/10.1016/j.jfoodeng.2009.08.018

13. Han, J.; Salmieri, S.; Tien, C. \& Lacroix, M. - J. Agr. Food Chem., 58, p.3125 (2010). PMid:20155911. http://dx.doi.org/10.1021/jf904443n
14. Assis, O. B. G. \& Silva, V. L. - Polímeros, 13, n.4, p.223, 2003. http:// dx.doi.org/10.1590/S0104-14282003000400006

15. Yoshida, C. M. P.; Oliveira-Junior, E. N. \& Franco, T. T. - Pack. Sci. Technol., 22, p.161 (2009). http://dx.doi.org/10.1002/pts.839

16. Yoshida, C. M. P.; Bastos, C.E.N. \& Franco, T.T. - Food Sci. Technol., 43, p.584 (2010).

17. Souza, B. W. S.; Cerqueira, M. A.; Martins, J. T.; Casariego, A.; Teixeira, J. A. \& Vicente, A. A. - Food Hydrocoll., 24, p.330 (2010). http://dx.doi.org/10.1016/j.foodhyd.2009.10.011

18. Farro, P. C. A.; Costa, G. P.; Sobral, P. J. A. \& Menegalli, F. C. Carbohydr. Polym., 81, p.838 (2010).

19. Henrique, C. M.; Teofilo, R. L.; Sabino, L.; Ferreira, M. M. C. \& Cereda, M. P. J. - Food Sci., 72, p.184 (2007). Pmid:17995770. http:// dx.doi.org/10.1111/j.1750-3841.2007.00342.x

20. Pellicano, M.; Pachekoski, W. \& Agnelli, J. A. M. - Polímeros, 19, n.3, p.212-217, 2009. http://dx.doi.org/10.1590/S010414282009000300009

21. Bergo, P. V. A.; Sobral, P. J. A. \& Prison, J. M. - J. Food Proc. Pres., 34, p.401 (2010). http://dx.doi.org/10.1111/j.1745-4549.2008.00282.x

22. Kechichian, V.; Ditchfield, C.; Veiga-Santos, P. \& Tadini, C. C. - Food Sci. Technol., 43, p.1088 (2010).

23. Denavi, G.; Blácido, D. T.; Añon, M. C.; Sobral, P. J. A.; Mauri, A. \& Menegalli, F. C. - J. Food Eng., 90, p.341 (2009). http://dx.doi. org/10.1016/j.jfoodeng.2008.07.001

24. Brandão, E. M \& Andrade, C. T. - Polímeros, 9, n.3, p.38 (1999).

25. Silva, M. A.; Bierhalz, A. C. K. \& Kieckbusch, T. G. - Carbohydr. Polym., 77, p.736 (2009). http://dx.doi.org/10.1016/j. carbpol.2009.02.014

26. Moura, M. R.; Rubira, A. F. \& Muniz, E. C. - Polímeros, 18, n.2, p. 132-137, 2008.

27. Shimoni, E.; Anderson, E. M. \& Labuza, T. P. - J. Food Sci., 66, p.1337 (2001). http://dx.doi.org/10.1111/j.1365-2621.2001.tb15211.x

28. Wanihsuksombat, C.; Hongtrakul, V. \& Suppakul, P. - J. Food Eng., 100, p.427 (2010). http://dx.doi.org/10.1016/j.jfoodeng.2010.04.027

29. Mai, N.; Audorff, H.; Reichstein, W.; Haarer, D.; Olafsdottir, G.; Bogason, S. G.; Kreyenschmidt, J. \& Arason, S. - Int. J. Food Sci. Technol., 46, p.297 (2011). http://dx.doi.org/10.1111/ j.1365-2621.2010.02475.x

30. Rodriguez-Amaya, D. B. - "Carotenoids and food preparation: the retention of provitamin A carotenoids in prepared, processed, and stored foods", J. Snow, Arlington (1997).

31. Brasil. - "Resolução n.44. Condições gerais de elaboração, classificação, apresentação, designação, composição e fatores essenciais de qualidade dos corantes empregados na produção de alimentos (e bebidas)". Brasília, 1977.

32. Notin, L.; Viton, C.; David, L.; Alcouffe, P.; Rochas, C. \& Domard, A. - Acta Biomater., 2, p.387 (2006). PMid:16765879. http://dx.doi. org/10.1016/j.actbio.2006.03.003

33. Associação Brasileira de Normas Técnicas - ABNT. ABNT NBR 5413. - "Iluminância de interiores". Rio de Janeiro, p.1, 1992.

34. Wyszecki, G. \& Stiles, W. S. - "Color science”, J. Wiley \& Sons, Inc., Nova Iorque (1967).

35. Gonçalves, E. M; Pinheiro, J.; Alegria, C.; Abreu, M.; Branda, T. R. S. \& Silva, C. L. M. - J. Agr. Food Chem., 57, p.5370 (2009). PMid:19863085. http://dx.doi.org/10.1021/jf901769w

36. American Society for Testing and Materials - ASTM. ASTM D882 “Tensile properties of thin plastic sheeting". Philadelphia, 1995.

37. Streit, N. M.; Canterle, L. P.; Canto, M. W. \& Hecktheuer, L. H. H. - Ciec. Rural, 35, p.748 (2005). http://dx.doi.org/10.1590/S010384782005000300043 
38. Volp, A. C. P.; Renhe, I. R. T. \& Stringuetta, P. C. - Aliment. Nutr., 20, p.157 (2009).

39. Olarte, C.; Sanz, S.; Echávarri, J. F. \& Ayala, F. - Food Sci. Technol., 42 , p.402 (2009).

40. Kasim, R. \& Kasim, M. U. - J. Food Agr. Environ., 5, p.126 (2007).

41. Tijskens, L. M. M.; Schijvens, E. P. H. M. \& Biekman, E. S. A. - Innov. Food Sci. Emerg. Technol., 2, p.303 (2001). http://dx.doi.org/10.1016/ S1466-8564(01)00045-5
42. Fernandes, S. C. M.; Freire, C. S. R.; Silvestre, A. J. D.; Desbrières, J.; Gandini, A. \& Pascoal Neto, C. - Ind. Eng. Chem. Res., 49, p.6432 (2010). http://dx.doi.org/10.1021/ie100573z

43. Bobbio, F. O. \& Bobbio, P. A. - "Introdução à química de alimentos", Varela, São Paulo (2003).

Enviado: 04/07/11

Aceito: $28 / 09 / 11$ 